\title{
Surface Magnetic Anomaly Triangulation Inversion
}

\author{
Jiaxiong Cai \\ Department of Land and Resources, Changsha, China \\ Email:827900498@qq.com
}

How to cite this paper: Cai, J.X. (2019) Surface Magnetic Anomaly Triangulation Inversion. International Journal of Geosciences, 10, 160-172.

https://doi.org/10.4236/ijg.2019.102010

Received: January 7, 2019

Accepted: February 16, 2019

Published: February 19, 2019

Copyright (c) 2019 by author(s) and Scientific Research Publishing Inc. This work is licensed under the Creative Commons Attribution International License (CC BY 4.0).

http://creativecommons.org/licenses/by/4.0/ (c) (i) Open Access

\begin{abstract}
Triangular system is a simplified magnetic anomaly interpretation system. In this paper, the expression of magnetic field of arbitrary independent shape model is given. Aiming at the undulating terrain, the technical support of "height tracking" and "bidirectional focusing" is designed to solve the influence of terrain on the interpretation process. The rule of triangulation of arbitrary independent magnetic anomaly inversion under undulating terrain is given, and the principle and procedure of accurate interpretation are given. The hypothesis of a single magnetic anomaly with arbitrary shape is presented. In this paper, an example is given to explain different parts of the same anomaly, and an example of prospecting in the Rosemary area of Peru is given. The system of the method in this paper is new ideas and advances in inversion of magnetic data under potential terrain.
\end{abstract}

\section{Keywords}

Graphic Transformation, Partition Law, Elevation Tracking, Bidirectional Focusing, Stepwise Solution

\section{Introduction}

Under the condition of horizontal terrain, the inversion formula of simple geometric magnet can be given by solving extreme value and inflection point [1] [2] [3], and under the condition of undulating terrain, no independent magnet can write the inversion formula related to extreme value and inflection point. It usually takes a lot of math. The magnetic field value on the undulating terrain can be approximately reduced to the magnetic field on the horizontal terrain by the treatment of "leveling", which can be used as the anomalous interpretation [4] [5] [6] [7] [8]. 
After more than 40 years of research on the triangle interpretation system of magnetic anomalies (referred to as "triangle system"), the reciprocal law of triangle system is found according to the principle of graph scaling, and the anomalies of all independent magnets of arbitrary shape under the condition of undulating terrain are found. A series of methods for interpreting individual magnetic anomalies without "leveling" are presented.

\section{Theoretical Basis for Inversion of Surface Magnetic Anomaly by Triangulation [9]-[15]}

The interpretation of surface magnetic anomaly by triangular system is based on the transformation of profile anomaly curve into conjugate triangle, and it relies on "height tracking" to resolve the influence of terrain as technical support. The reciprocal law of countdown is found even in the undulating terrain. The reciprocal law is an important theoretical basis for the accurate inversion of arbitrary shape independent magnets under undulating terrain conditions.

\subsection{Model Foundation}

Under the condition of undulating terrain, the magnetic field expressions of lenticular, platelike, garlic and other forms can be written as follows:

$$
\begin{gathered}
\Delta T_{x y}=\frac{1}{n} \sum_{i=1}^{n} 2 b_{i} J_{S} \sin \beta \sqrt{1-\cos ^{2} I_{0} \cos ^{2} A} F_{i}\left(h_{x y} x, y \pm L_{i}, l_{i}\right) \\
F_{i}\left(h_{x y}, x, y \pm L_{i}, l_{i}\right)=f\left(h_{x y}, x, y+L_{i}, l_{i}\right)-f\left(h_{x y}, x, y-L_{i}, l_{i}\right) \\
f\left(h_{x y}, x, y+L\right) \\
=\frac{L_{z}}{\sqrt{c^{2}+L_{z}^{2}}} \frac{(x-l \cos \beta) \cos \xi-\left(h_{x y}+l \sin \beta\right) \cos \xi}{(x-l \cos \beta)^{2}+\left(h_{x y}+l \sin \beta\right)^{2}}-\frac{L_{z}\left(x \sin \xi-h_{x y} \cos \xi\right)}{\sqrt{\left(D^{2}+L^{2}\right)} D^{2}} \\
-\frac{1}{\left(B^{2}+L_{z}^{2}\right)}\left(\frac{P+1}{\sqrt{c^{2}+L_{z}^{2}}}-\frac{P}{\sqrt{D^{2}+L_{z}^{2}}}\right)\left(\left(\cos \xi \cos \beta-\cos \xi-\cos ^{2} \xi+K^{2}\right) L_{z}\right. \\
\left.-2 k \sin \left(i_{s}-\beta\right) B\right)+2 K \cos \left(i_{s}-\beta\right)\left(\frac{1}{\sqrt{c^{2}+L_{z}^{2}}}-\frac{1}{\sqrt{D^{2}+L_{z}^{2}}}\right)
\end{gathered}
$$

where: $L_{z}=Y+L ; \quad L_{f}=Y-L ; \quad P=h_{x y} \sin \beta-x \cos \beta ; \quad B=x \sin \beta+h_{x y} \cos \beta$; $D^{2}=x^{2}+h_{x y}^{2} ; c^{2}=(x-l \cos \beta)^{2}+\left(h_{x y}+l \sin \beta\right)^{2} ; K=\cos i_{s} \operatorname{ctg} A ;$

$i_{s}=\operatorname{arctg}\left(\operatorname{tg} I_{0} \csc A\right) ; \quad h_{x y}=h+H(x, y) ; b_{i}=b_{1}, b_{2}, b_{3}, \cdots, b_{n}$;

$L_{i}=L_{1}, L_{2}, L_{3}, \cdots, L_{n} ; l_{i}=l_{1}, l_{2}, l_{3}, \cdots, l_{n} ; H(x, y)$ For height data with zero origin; $\xi=2 i_{s}-\beta$ Magnetization characteristic angle; $L$ Half length of the direction of the magnetic source; 1 Magnetic source extension; $b$ Half thickness of magnetic source; $\beta$ Magnetic source inclination; $I_{0}$ Geomagnetic inclination; $i_{s}$ in section magnetization characteristic dip; $A$ Magnetic source azimuth; $J_{s}$ Magnetization in the section.

1) The formula is the model foundation of triangle system. The "variable 
model", which can make arbitrary independent morphologic transformation, reserves numerous methods for explaining the anomalies of the independent form magnets for the triangular system.

There are as many as 7 variables related to magnets in formula (1), which cannot give a general algebraic solution under normal conditions.

In this paper, we choose a series o schemes to solve the problem step by step, starting with the virtual interpretation, graphic transformation combining with the progressive correction of the abnormal plane information after the graph transformation from the profile anomaly.

\subsection{Graphic Transformation}

In the case of oblique magnetization, the profile anomalies have the characteristics of positive and negative concomitant curves. "graphic conversion" refers to the maximum slope tangent based on the abnormal curve (S1, S2, S3) Transform into conjugate triangles.

Conjugated triangles from regular triangles " $\triangle A U V$ ” And inverted triangle “ $\nabla V D E$ " Composition (Figure 1).

Add two vertical lines to two triangles. The underside of a cut triangle is " $b$ " and " $c$ "; partitioning. The bottom of the inverted triangle is " $e$ " and " $d$ ". Thus, To two groups of "conjugate ratios":

$$
\left[\begin{array}{l}
K_{A}=\frac{a-b}{a+b} \\
K_{v}=\frac{d-e}{d+e}
\end{array}\right]
$$

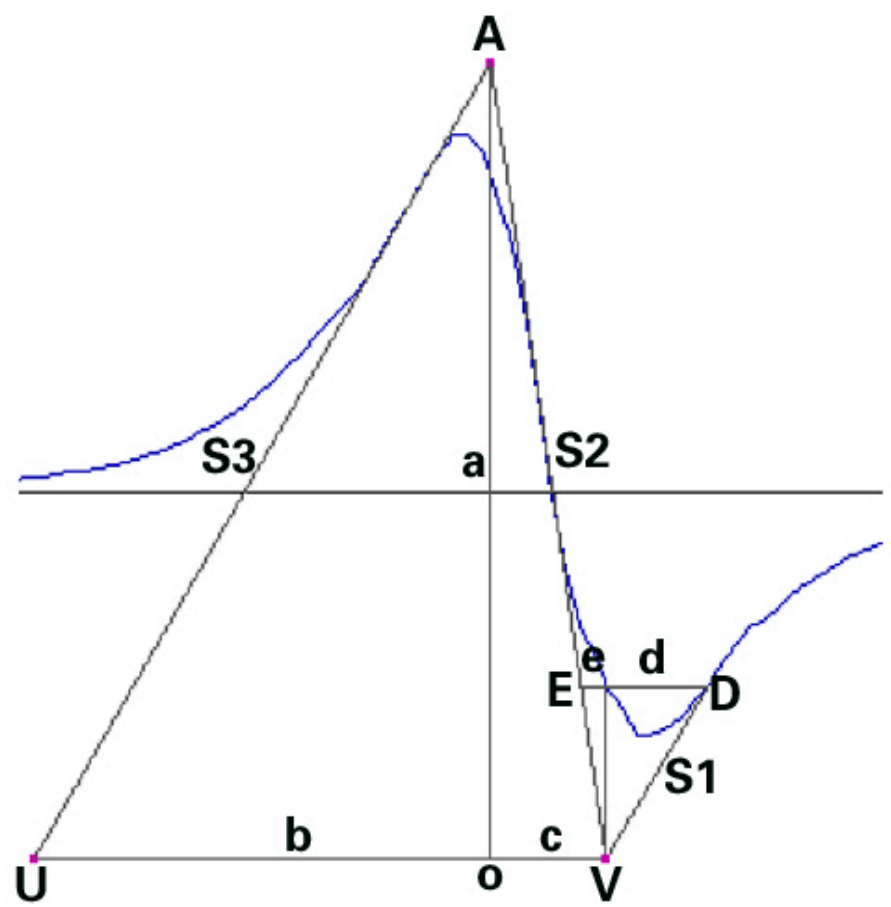

Figure 1. Graphic transformation. 
The conjugate ratio of the original anomaly intersects at one point in a rectangular coordinate system, called Conjugate points. Conjugate points are the objects to be pursued by interpreting anomalies with ordered changes, obtaining conjugate ratios and crossing them. Once the conjugate point and the intersection point of the interpretation anomaly coincide, the conjugate triangle of the original anomaly and the conjugate triangle of the interpretation anomaly will become "similar conjugate triangles", and the relationship between them will become a scaling relation.

\subsection{Height Tracking}

Setting the origin height of the interpretation anomaly to "0", and setting the height of each point in the interpretation profile, must be taken as the unit of the top buried depth of the magnet, which can be obtained one by one during the interpretation process.

$$
\left[\begin{array}{c}
O_{h}=0 \\
H(i)_{j}=\frac{H(i)}{h_{J}}
\end{array}\right]
$$

In the form, $O_{h}:$ It is the relative elevation of the origin; $H(i)_{j}$ : The array of heights used to explain points in a section in height; $H(i)$ : An array of section heights with zero height at the origin of the anomaly; $h_{J}$ : The top burying depth of the magnet was obtained by the successive interpretation during the interpretation process.

It can be seen that height tracking can correctly contain the actual influence of topography on anomalies when the anomaly changes in the interpretation process.

\subsection{Partition Law}

Under the condition of horizontal terrain, simple independent magnets, such as infinite extended thin plate, infinite lengthened horizontal cylinder and sphere, can give the general algebraic solution and construct the inversion formula on the premise that the equation is less than 5 degrees. However, for the lens body, thick plate body, garlic body, tip body and other independent magnets anomaly, plus in the undulating terrain conditions, its anomaly is unable to give a general algebraic solution.

By using the isobaric relation between similar conjugate triangles, it is found that the reciprocal of the bottom edges of a triangle is the first algebraic solution of (1) innumerable variables, and the bottleneck problem without solution is solved. For this reason, the relation between the inverse of the bottom edge and the solution is called the Partition law. The Partition law is the theoretical basis of the triangulation inversion of arbitrary shape independent magnetic source under the condition of undulating terrain.

The buried depth of magnets on arbitrary independent magnets under the condition of undulating topography $(h)$ General inversion formula: 


$$
h=\frac{1}{U V}(b+c)
$$

Intermediate reciprocal “ $\frac{1}{U V}$ ” Is a similar conjugate triangle in interpretation $U V$ The reciprocal of two point coordinates; $(b+c)$ : In a conjugate triangle transformed by the original abnormal graph "regular triangle". The bottom edge is divided by the vertical foot of the vertex.

In fact, (4) is the ratio of the corresponding underside of the two similar triangles before and after interpretation. Since the top depth of the magnet is set as a scale unit in the process of interpretation, when the conjugate triangle before and after interpretation satisfies the similarity relation, it also satisfies the scaling relationship of the graph. The ratio between them eliminates the influence of most variables and only leaves the relation between the top depth of the magnet and the ratio.

In horizontal terrain, simple geometric magnets, $\frac{1}{U V}$ All have explicit function composition. If the board body is infinite:

$$
\frac{1}{U V}=\left[\frac{\sqrt{3} \cos \frac{\xi}{3}-\sin \frac{\xi}{3}}{\sqrt{3} \cos \frac{\xi}{3}+\sin \frac{\xi}{3}}-\frac{8\left(\sqrt{3} \cos \frac{\xi}{3}+\sin \frac{\xi}{3}\right)^{2} \sin ^{2} \frac{\xi}{3}}{\left(\sqrt{3} \cos \frac{\xi}{3}-\sqrt{3} \sin \frac{\xi}{3}\right)^{3}\left(\cos \frac{\xi}{3}+\sqrt{3} \sin \frac{\xi}{3}\right)}\right]
$$

It can be seen from the analysis that the reciprocal system is composed of the pure ratio of the characteristic angle function of magnetization in the section, which eliminates the influence of the length of the magnet, the extension of the lower part and the magnetic moment and so on. Other simple geometric magnets all have similar reciprocal solutions, and the difference is different in complexity; the common point is that the influence outside the angle is eliminated.

The inversion formula of reciprocal law has only ratio relation and is suitable for all independent geometric form magnets. The theoretical value of the reciprocal law lies in the fact that the triangulation can solve the inversion problem of any independent shape magnet anomaly under the undulating terrain.

\subsection{Two-Way Focusing}

The triangulation can get the accurate explanation result. Because of the interpretation system embedded in the "two-way focus" coordination interpretation function (Figure 2).

In the triangulation, the anomaly obtains the accurate explanation essential factor, first is the origin localization must be accurate. The second is the exact depth of the magnet vertex.

Bidirectional focusing refers to the transverse focusing of the abnormal origin in the undulating terrain profile, and the vertical focus of the magnet vertex is realized by interpreting the step size.

Under the condition of oblique magnetization, the origin of the profile 


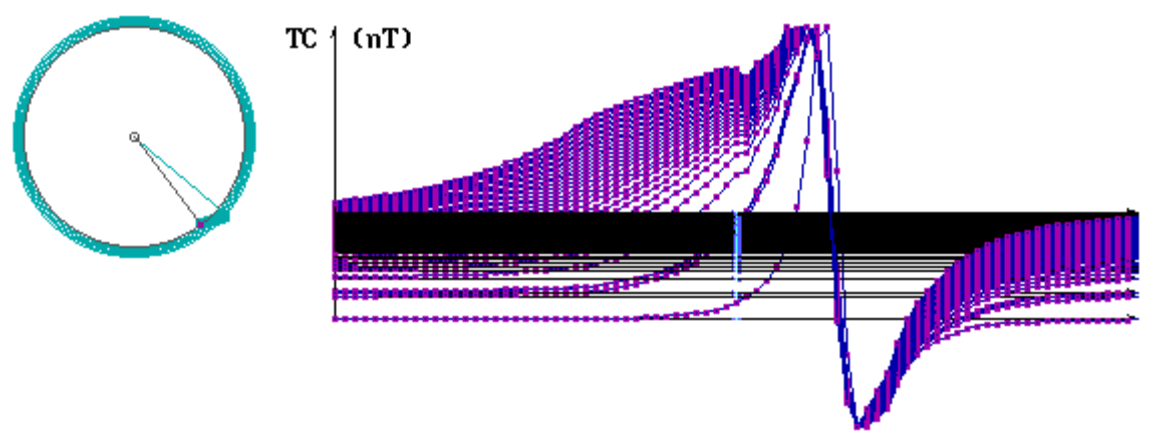

Figure 2. The process between the origin and the vertex of the magnetic source is interpreted harmoniously by bidirectional focusing [2]. When the two pointers in the explanation clock on the left side coincide, it is shown that the vertex of the conjugate triangle system is overlapped, and the location of the origin is indirectly determined, and the location of the magnet vertex is indicated when the pointer length and the length of the same end point are coincident. On the right is the family of real-time interpretation curves in the double focusing process.

anomaly lies between the positive extreme value and the negative extreme value of the abnormal curve, but it is difficult to determine the specific position. When the profile anomaly is transformed into a conjugate triangle by graph, the origin of the anomaly is between A and V. The focus of the vertex of the conjugate triangle before and after interpretation is replaced by the focus of the anomaly origin. The process of focusing the origin on the undulating terrain can be expressed as follows:

$$
F o \Rightarrow F_{O}-\frac{A_{X}-A_{O X}}{2} \Rightarrow 0
$$

Type center Fo In order to explain the coordinate distance between the vertex of abnormal conjugate triangle and the vertex of original conjugate triangle; $A_{X}$ In order to explain the vertex coordinates of abnormal conjugate triangle; $A_{O X}$ Is the vertex coordinates of the original anomalous conjugate triangle.

The formula represents the principle and process of gradual focusing of vertex spacing of conjugate triangles in the interpretation of triangular systems.

The conjugate triangle obtained from the original anomaly has an approximate relation between the bottom side and the top depth of the magnet:

$$
h_{x} \approx \frac{b+c}{\sqrt{2 \pi \sin A+\frac{8}{\pi}}}
$$

Type center $A$ Magnet strike.

The initial interpretation step size is given according to the above formula, and the interpretation step size in the triangulation interpretation process changes with the interpretation process. When the explanatory step size is changed to "focus step", the depth of top burying on the magnet is an exact solution. By explaining the process of approaching the focusing step by step size, that is, the focusing process of the triangle system in the vertical direction of the section. 
Focusing step $F_{X}$ exact solution of buried depth on top of magnet $h$. The relationship is:

$$
F_{X}=\frac{D}{n h}
$$

Type center $D$ to explain the length of the section; $n$ to explain the number of points in a section.

The coordinated realization of trigonometric bidirectional focusing is an important technical support for accurate interpretation results.

\subsection{Step by Step Solution}

On the basis of setting scale, unit of intensity and virtual individual unknown parameters, the interpretation process of triangle system is to change two key parameters, to construct similar conjugate triangles, to give preliminary interpretation results by applying "Partition law", and to modify virtual parameters. Complete the exact interpretation step by step.

\subsubsection{Interpretation of Virtual Identification}

The anomalous section of any position and direction is chosen as the interpretation section. The shape of the magnetic source and the parameters of the magnetic source are unknown. Therefore, it is assumed that the anomaly is caused by the bookboard, and the following steps are used to give a set of explanation results including the identifiable parameters.

In order to solve the problem by using the simplified pair (1) formula, seven parameter variables related to the independent magnet are obtained ( $M_{S}, h, L, l, \xi, \beta, b$ ), According to the difficulty of solving the problem, it is dealt with step by step. Set two units of interpretation: set the top depth of the magnet as a unit of scale ( $\left.h_{0}=1\right)$; According to the difficulty of solving the problem, it is dealt with step by step. Set two units of interpretation: set the top depth of the magnet as a unit of scale $\left(M_{S 0}=1\right)$; The ratio of the strike half length of the virtual magnet to the buried depth of the top of the magnet is equivalent to the ratio of the long axis to the long axis in the magnetic anomaly plane diagram. ( $L_{0}=h \frac{A_{\max }}{A_{\text {nin }}}$, meantime $h=1$ ); $b$ : the half thickness of magnet should be determined by thickening after shape recognition. $\xi$ : The characteristic angle of magnetization in the section and the extension of the magnet in the section (I). It is obtained by interpreting a similar conjugate triangle.

Order change $\xi, l_{X}$ Two parameters, The preferred method for shape determination in triangles is the book plate interpretation method. After the differential integral discriminant parameter of the profile curve determines the shape of the independent magnetic source in the section, the semi-thickness and the general shape of the magnetic source can be obtained by thickening judgment [16].

On the basis of formula (1), there is an interpretation method corresponding to any independent magnetic source. After obtaining the similar conjugate tri- 
angle, the top depth of the magnetic source is obtained according to the Partition law, and the following results are given one after another:

$$
\text { Magnetic moment } M_{S}=h \frac{1}{A o * U V} a(b+c)
$$

In the form, $A o * U V:$ It is the product of the vertex coordinate and the bottom coordinate. $a(b+c)$ : It is the product of "height" and "bottom edge" of the peak triangle after the transformation of abnormal section figures. The first is reciprocal and the latter is the ratio.

Magnetic source down $l=h l_{X}$

Magnet inclination $\beta=2 i-\xi$

After the original anomaly is explained by the plate of the triangle system, according to the identification parameters given by the differential and integral processing function of the curve inlaid in the program, it can be determined that the magnet corresponding to the abnormal section extends thicker and thinner. The change trend of equal thickness (as shown in Figure 3 is the trend of pinnacle [16]. Thus, the shape of magnetic source corresponding to the profile anomaly is determined.

\subsubsection{Thickening Amendment Interpretation}

After the initial shape of the magnetic source is determined, through the ordered thickening of the magnet, the virtual parameters are corrected by comparing the interpretation results with the plane diagram.

When the identification parameter is changed, it is shown that the thickening is excessive, and when the remaining anomaly is reversed, the virtual parameter is corrected too much. explanatory text "Tc"- $\Delta \mathrm{T}$; QM2018fy; program name; QJF15WF8 Section name; PD Offset points; MX model; JS explain; PW Offset number; PJ Bias angle; MAX maximum; MIN minimum; Ms Effective magnetization direction in section; Red dashed line Residual anomaly.

\subsubsection{Precise Interpretation}

When the remaining anomalies in the interpretation result section completely

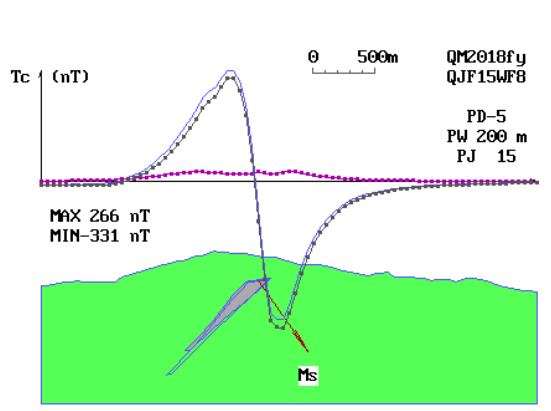

(a)

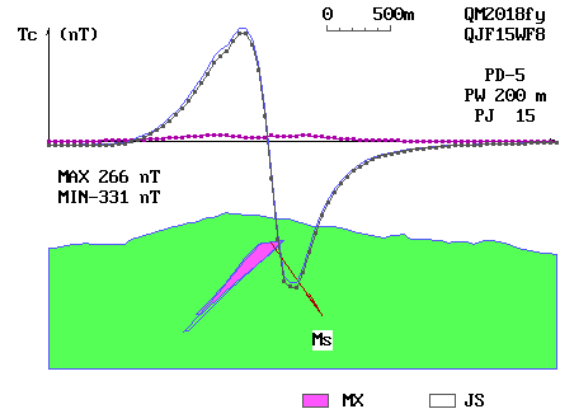

(b)

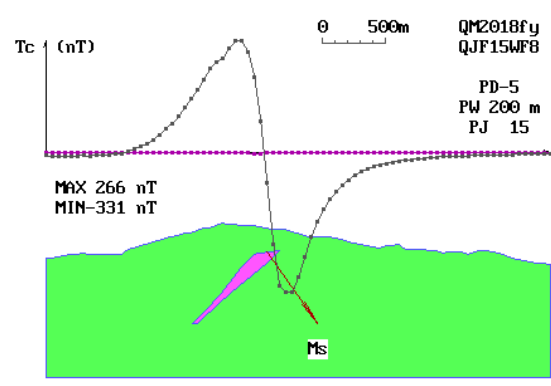

(c)

Figure 3. Curved surface magnetic anomaly triangulation inversion step by step to solve sequence profile. (a) Virtual recognition. (b) Thickening correction. (c) Precise interpretation. 
coincide with the abscissa line and the interpretation result plane and the original anomaly plane completely coincide, the inversion result given by the triangle system is the accurate interpretation result. The relative error of the accurate interpretation result can be ensured to be less than $0.1 \%$.

In practice, as long as there is no obvious residual anomaly in the interpretation result profile, the interpretation result can be regarded as the usable result when the interpretation results.

The cooperative realization of bidirectional focusing is an important technical support for accurate interpretation.

The step-by-step technique results are shown in Table 1.

\section{Trial Calculation}

In the undulating ground, two different positions and different directions are chosen, which are located within an independent anomaly (Figure 4).

The shape of undulating topography in the section is different, the abnormal characteristics are different, and the abnormal amplitude is different. The triangulation results show that the interpretation results have the same accuracy (Figure 5, Table 2).

\section{Living Example}

Peru verifies that under the condition of demagnetization and inclined topography, the triangle system interpretation has achieved good ore-finding effect.

Prior to verification, the triangular system inferred that the magnet appeared at 290 meters in No. 1 of line 18, and the inclination of the magnet was 45 degrees. Hole inspection in $286-297$ meters see 11 meters of magnets rich ore, ore dip angle 46 degrees (Figure 6 \& Figure 7).

Based on the results of ore discovery in hole 1, the geophysical prospecting has been confirmed by the subsequent station exploration, and it is expected that the anomalous area will become at least a medium magnet-rich deposit.

Table 1. Summary of the results of Triangle system step by step solution.

\begin{tabular}{|c|c|c|c|c|c|c|c|}
\hline $\begin{array}{c}\text { Triangular } \\
\text { system }\end{array}$ & $\begin{array}{c}\text { Section } \\
\text { magnetic } \\
\text { moment } \\
M_{s}\left(\mathrm{~A} \cdot \mathrm{m}^{2}\right)\end{array}$ & $\begin{array}{c}\text { Top } \\
\text { burying } \\
\text { depth } \\
h(\mathrm{~m})\end{array}$ & $\begin{array}{l}\text { Magnet } \\
\text { length } \\
2 L(\mathrm{~m})\end{array}$ & $\begin{array}{c}\text { Lower } \\
\text { extension } \\
I(\mathrm{~m})\end{array}$ & $\begin{array}{c}\text { Magnet } \\
\text { inclination } \\
\beta\left(^{\circ}\right)\end{array}$ & $\begin{array}{c}\text { Total } \\
\text { magnetic } \\
\text { angle } \\
\xi\left(^{\circ}\right)\end{array}$ & $\begin{array}{l}\text { Magnet } \\
\text { thickness } \\
2 b(\mathrm{~m})\end{array}$ \\
\hline design & 8000 & 200 & 2000 & 800 & 135 & 154.4 & 200 \\
\hline $\begin{array}{c}\text { Virtual } \\
\text { recognition }\end{array}$ & 8458 & 208.8 & 1462 & 1042.5 & 133.7 & 155.7 & 10 \\
\hline $\begin{array}{l}\text { Thickening } \\
\text { correction }\end{array}$ & 8174 & 204.5 & 1595.4 & 966 & 133.8 & 155.6 & 180 \\
\hline $\begin{array}{c}\text { Precise } \\
\text { interpretation }\end{array}$ & 7999.9 & 199.99 & 1999.9 & 799.9 & 135 & 154.4 & 199.9 \\
\hline
\end{tabular}




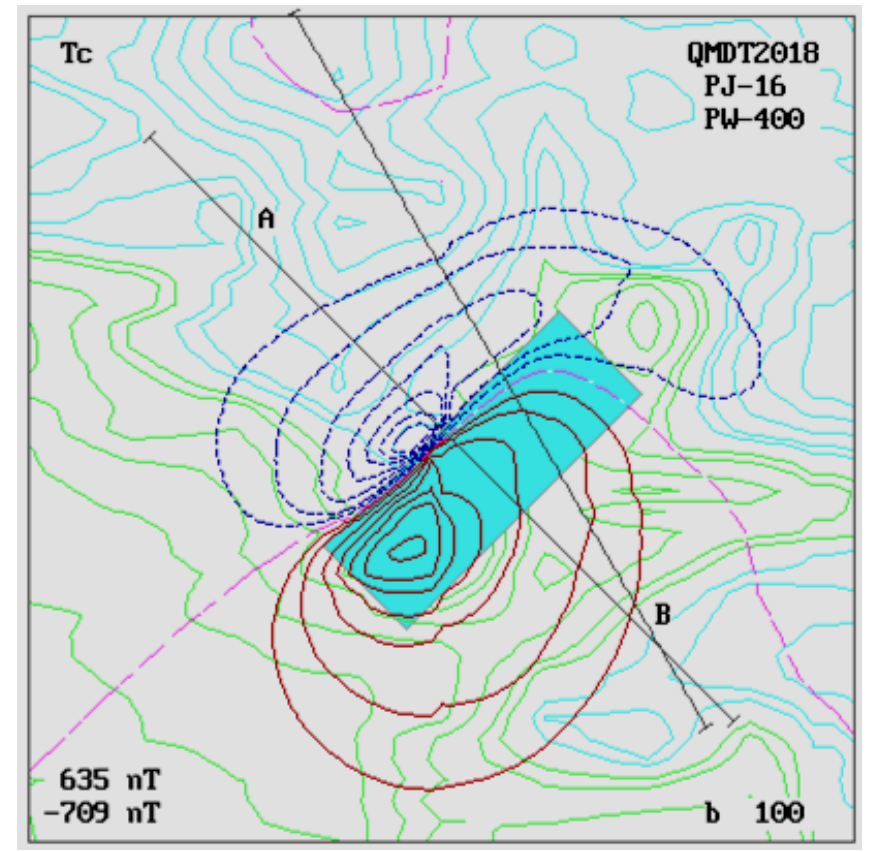

Figure 4. Two interpretation profiles in different directions.

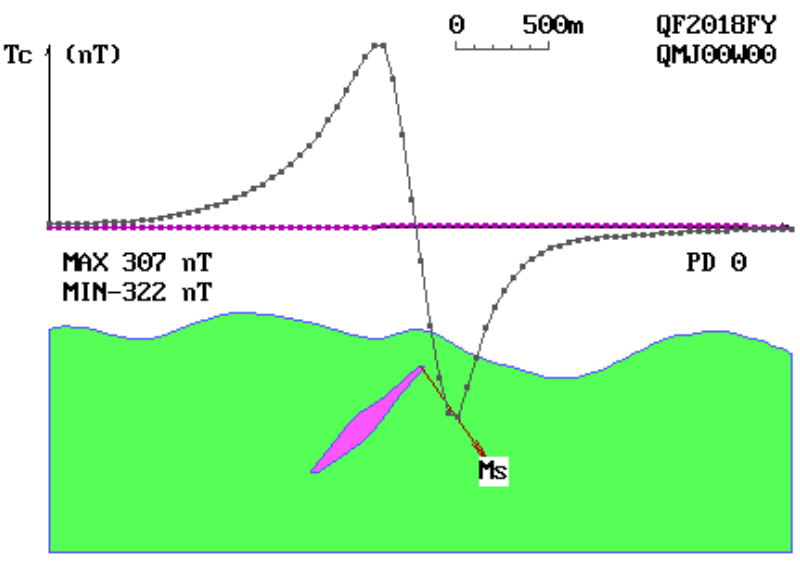

(a)

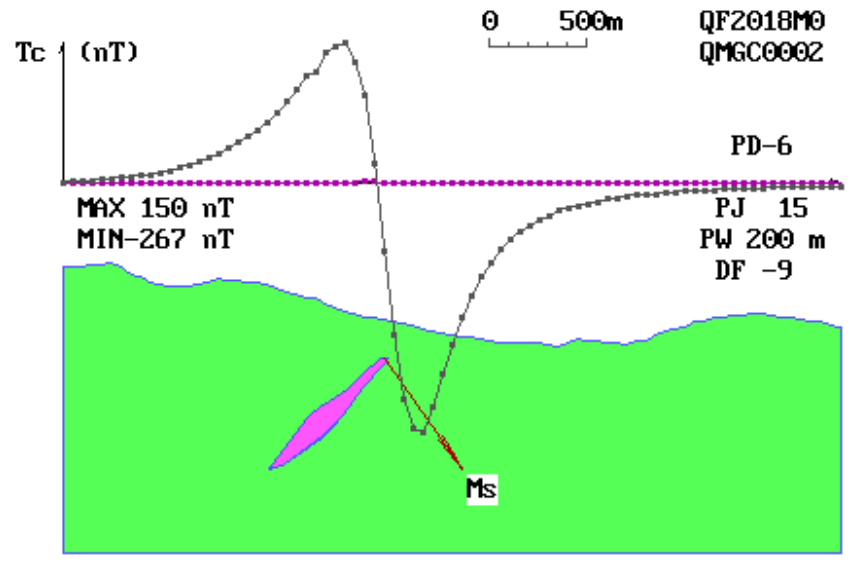

(b)

Figure 5. Interpretation results of magnetic anomaly profiles at different locations and directions Map note DF magnet top dip angle.

Table 2. Summary of results of magnetic anomalies in triangular interpretation of different positions and directions.

\begin{tabular}{crrrrrrrr}
\hline$\Delta$ & $M_{s}$ & $h$ & $2 L$ & 1 & $\beta$ & $2 b$ & 135 & 154.4 \\
design & 8000 & 200 & 1600 & 800 & 800.1 & 134.9 & 154.4 \\
A & 8001 & 200 & 1602 & 160.9 & 135 & 154.4 \\
B & 8001 & 200 & 1604 & & 200 \\
\hline
\end{tabular}

\section{Conclusions}

In the triangulation inversion of surface magnetic anomaly, the discovery of Partition law breaks through the bottleneck problem of surface magnetic anomaly interpretation. 
$\mathrm{Bi}$-directional focusing is the technical support for surface magnetic anomaly inversion to achieve accurate interpretation level.

The "Partition law" found in the fusion of graphic transformation, height tracking, bidirectional focusing and similarity solving is not only a theoretical development in inversion of magnetic anomalies to overcome the influence of geodesic topography and accurately interpret the related parameters of magnetic sources, but also in gravity. In the research of electromagnetic inversion, it can be used for reference to overcome the influence of terrain.

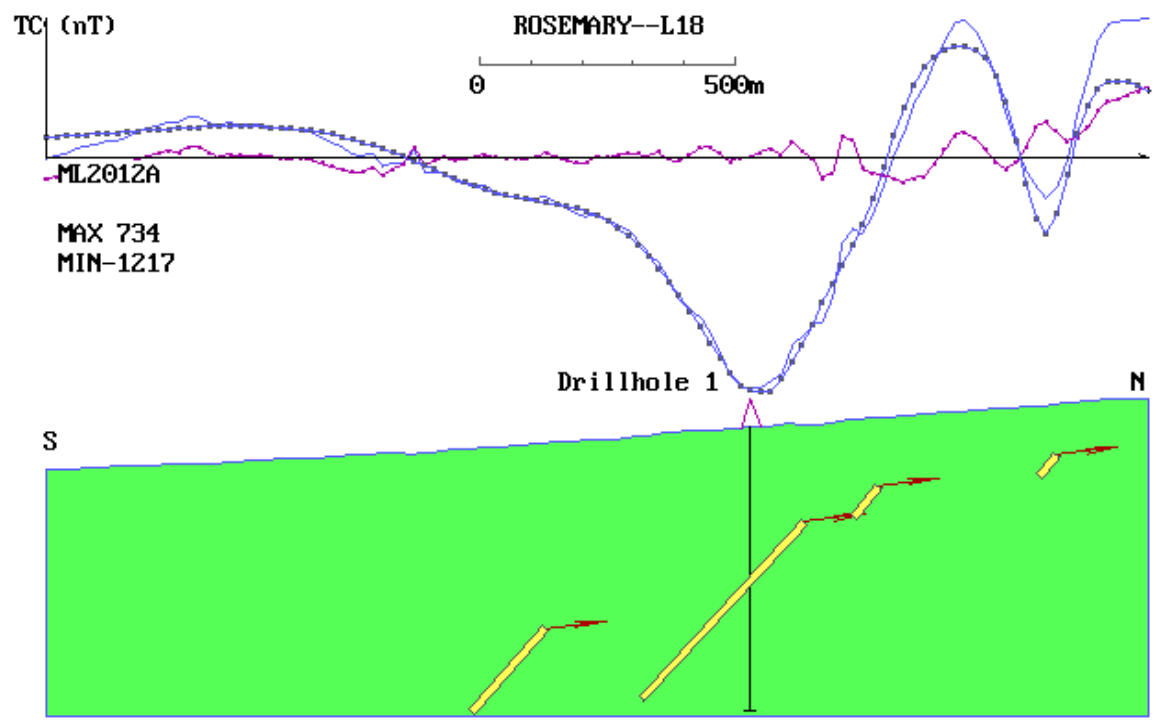

Figure 6. ROSEMARY magnetic anomaly triangulation interpretation profile.
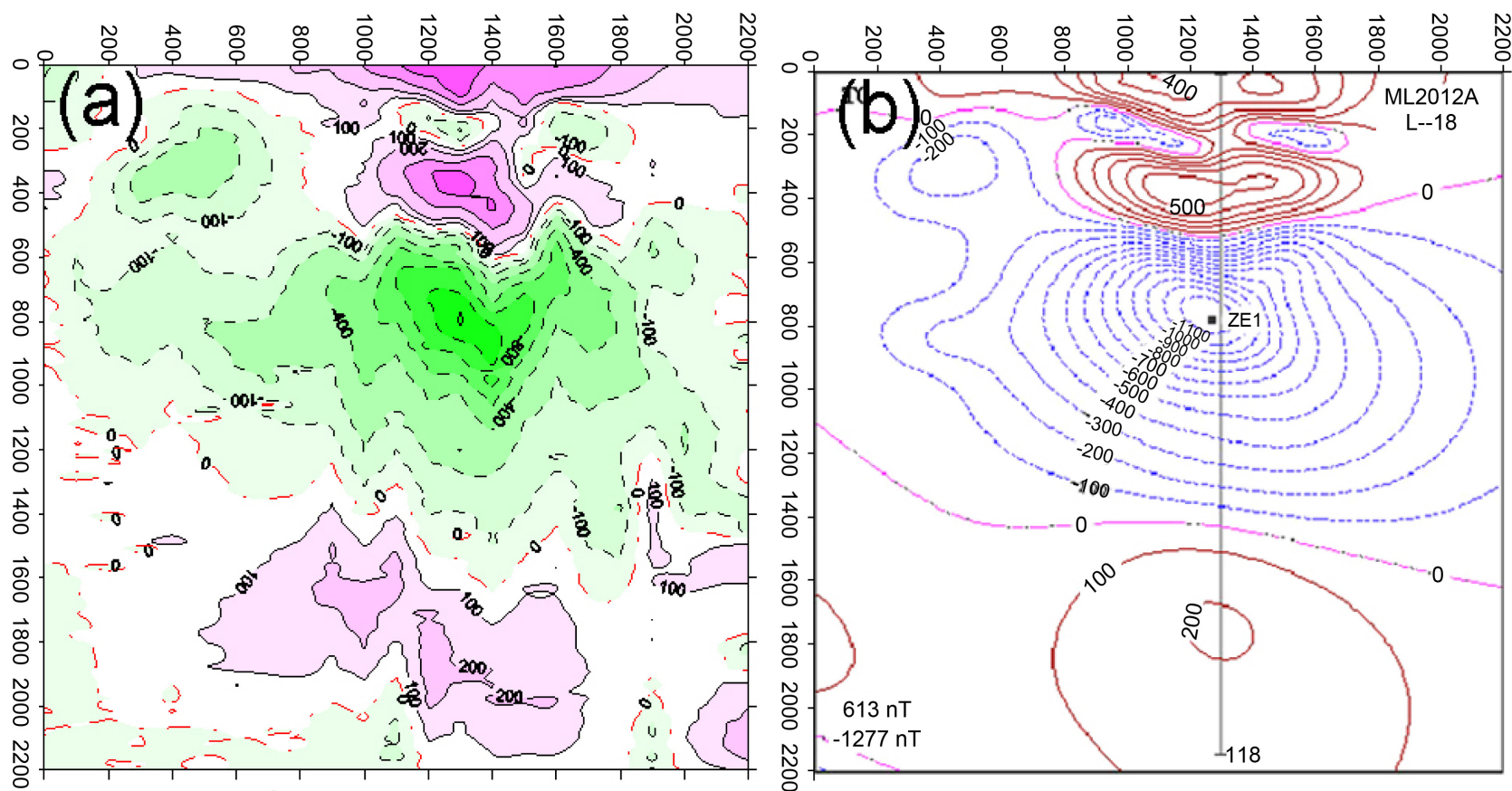

Figure 7. ROSEMARY measured magnetic anomaly (a) and triangulation interpretation result Magnetic anomaly (b) contrast plane diagram. 
In theory, the triangle system can distinguish the shape of any independent magnet under the condition of undulating terrain by virtual recognition, thickening correction and accurate interpretation, and can give the relevant parameters of the magnet accurately. It is often difficult to do it perfectly because of the influence of the superposition of anomalies and other factors. One step that can be determined is virtual interpretation, and the other parameters except the thickness of the magnet are calculated by the method of the sheet plate. In general, as long as the residual anomaly is not obvious, the result of interpretation is still of practical significance in the virtual interpretation section, and the example of Peru prospecting in this paper is a good example.

\section{Acknowledgements}

Thank you Mr. Zhao Shuangqiu for providing the magnetic data of Peru and completing the inference and interpretation work together.

\section{Conflicts of Interest}

The author declares no conflicts of interest regarding the publication of this paper.

\section{References}

[1] Cai, J.X. (1989) Magnetic Anomaly Triangle Interpretation System. Geological Publishing House, Beijing, 12-21.

[2] Cai, J.X. and Zhao, S.Q. (2017) Interpretation of Magnetic Anomalies of Thin Plates under Undulating Terrain. China Science and Technology, 10, 1416-1424.

[3] Gramt, F.S. (1965) The Quantitative Interpretation of Magnetic Anomalies. Interpretation Theory McGraw-Hill Book Company, 340-350.

[4] Hou, C.-C., Cai, Z.X. and Liu, K.-J. (1985) Setting up the Interpretation System of Potential Field Conversion on Curved Surface from Even Layer. Journal of Geophysics, 28, 76-84.

[5] Yao, C.L. and Huang, W.N. (1997) Combined Use of the Potential Field and Its Vertical Gradient Fast Spline Curve Leveling Method. Petroleum Geophysical Prospecting, 32, 229-236.

[6] Yao, C.L. and Guan, Z.N. (1997) Comprehensive Utilization of Total Field Anomaly and its Fast Quoving Method of Vertical Gradient. Modern Geology, No. 1, 78-83.

[7] An, Y.L., Chai, Y.P., Zhang, M.H., Jin, M., Qiao, S.H. and Qu, H.P. (2013) Using the Best Equivalent Source Model and a New Method to Derive the Expression of Unit Potential Field. Journal of Geophysics, No. 7, 2473-2483.

[8] Liu, J.L., Wang, W.Y. and Yu, C.C. (2007) Study on the Method of Approaching Quhua Ping Step by Step. Journal of Geophysics, 50, 1351-1357.

[9] Cai, J.X., San, Y. and Qu, H.P. (1994) Magnetic Anomalies. Journal of Central South Institute of Mining and Metallurgy, 25, 631-632.

[10] Yan, Y.P., Cai, J.X. and Dai, Q.W. (2014) Conjugate Interpretation Theory and Method of Magnetic Anomaly. Geology Press, Beijing, 89-91.

[11] Liu, X.Z. (1993) Principles and Applications of Gravity and Magnetic Anomaly Spectrum Analysis. Chongqing Publishing House, Beijing, 132-167. 
[12] Cai, J.X. (1990) A Method of Magnetic Anomaly Decomposition Inversion and Distortion Correction, Collection of Computational Geophysical Studies. Seismological Press, Beijing, 279-288.

[13] Cai, J.X., Li, Y. and Dai, Q.W. (2016) Research on the Source of Magnetic Anomaly Field. China Science and Technology Paper Online.

[14] Yan, Y.P., Dai, Y. and Cai, J.X. (2014) Theory and Method of Conjugate Interpretation of Magnetic Anomalies. Geological Publishing House, 89-91.

[15] Yan, Y.P., Dai, Q.W., Cai, J.X. and Lu, G.M. (2011) Magnetic Anomaly Conjugate Element Inverse Interpretation Method. Journal of Central South University, Natural Science Edition, 731-737.

[16] Cai, J.X. (2018) Differential Integrals of Curves and Inversion of Magnetic Source Morphology. Journal of Applied Mathematics and Physics, 6, 1160-1169. 